\title{
SPECTROSCOPY OF RADIO SOURCES FROM THE THIRD BOLOGNA SURVEY
}

\author{
S. DJORGOVSKI* AND D. J. THOMPSON
}

Palomar Observatory, MS 105-24, California Institute of Technology, Pasadena, California 91125

AND

M. VIGOTTI AND G. GRUEFF $\dagger$

Instituto di Radioastronomia C.N.R., Via Irnerio 46, I-40126 Bologna, Italy

Received 1989 October 27

\begin{abstract}
We present long-slit CCD spectroscopy of eleven optical candidates for selected radio sources from the third Bologna survey (B3). One object $(0141+398)$ is a probable misidentification, a foreground star. The remaining ones are a mixture of quasars and active galaxies, ranging in redshift from 0.11 to 0.85 . B3 $0219+443$ is a steep-spectrum quasar. B3 $1141+374$ is identified with an interacting galaxy pair, with the southern component harboring an active nucleus.
\end{abstract}

Key words: galaxies: redshifts-quasars-redshifts-spectroscopy

The third Bologna radio survey (B3; Ficarra, Grueff, and Tomassetti 1985) consists of a complete sample of 13,354 sources brighter than $0.1 \mathrm{Jy}$ at $408 \mathrm{MHz}$, in the interval $37^{\circ} 15^{\prime}<\delta<47^{\circ} 37^{\prime}$. A subset of 1103 sources in five flux bins was defined by narrowing the survey strip in $\delta$ and restricting it to high galactic latitudes. VLA maps were then obtained for all sources in this subsample (B3VLA). More details were given by Vigotti et al. (1989). The survey spans an intermediate range in radio flux (from $0.1 \mathrm{Jy}$ up, at $408 \mathrm{MHz}$ ), thus filling the gap between the 3CR and other high-flux samples and the mJy-level samples. About 400 sources have optical identifications proposed so far, but only $\sim 100$ of them have measured redshifts.

We are conducting a program of optical identifications and spectroscopy of the B3VLA sources at Palomar Observatory and elsewhere. Our purpose is to form a complete sample of moderate-power radio sources at cosmologically interesting redshifts, for studies of galaxy evolution and radio source counts, evolution of radio source properties (e.g., linear size, morphology) with redshift, comparisons of galaxies with a wide range of radio power at similar redshifts, a search for extremely distant clusters of galaxies, steep-spectrum quasars, other unusual types of objects, and so on. Here we present the spectroscopic results on some of the higher objects observed to date at Palomar. The radio information and optical identifications proposed for the sources observed in this work were published by Vigotti et al. (1989).

\footnotetext{
*Alfred P. Sloan Foundation fellow.

$\dagger$ Also at Dipartimento di Astronomia, Università Degli Studi di Bologna, Italy.
}

The observations reported here were done on UT 1988 January 10 and 11 and March 10 and 11 using the Double Spectrograph (Oke and Gunn 1982) mounted at the Cassegrain focus on the Hale 200-inch (5-m) telescope. The conditions were mostly nonphotometric, with variable transparency, and the seeing was never better than about 2 arc sec (FWHM). The slit width used was 2 arc sec and the effective spectral resolution (FWHM) was about $10 \AA$. The wavelength range covered was different for different nights, but typically was $3700 \AA-7400 \AA$ or $3600 \AA-9000 \AA$; on UT 1988 January 10 there was a gap of about $500 \AA$ in the coverage between the blue and the red sides of the spectrograph. The exposure times were in the range of 600-2500 sec. Exposures of arc lamps were used to obtain the dispersion solutions, giving a wavelength calibration rms of $\sim 1 \AA$. Dome flat fields and exposures of standard stars from Oke and Gunn (1983) were used to correct for the instrumental response and to provide a crude flux calibration, and we estimate the zero-point uncertainties to be of the order of 0.5 . The data were reduced using standard procedures.

A total of eleven sources were observed, of which two turned out to be quasars, five narrow emission-line galaxies, two galaxies with a weak or absent line emission, one apparently interacting galaxy pair, and one galactic star (probably a misidentification of B3 $0141+398$, even though the optical and radio coordinates differ by only 2-3 arc sec). Some representative spectra are shown in Figure 1. Table 1 lists the information on measured lines and redshifts. In addition to the features listed there, other absorption (e.g., the $4000 \AA$ break) or emission (e.g., the [S II] $\lambda \lambda 6716+6731$ doublet) were often seen 

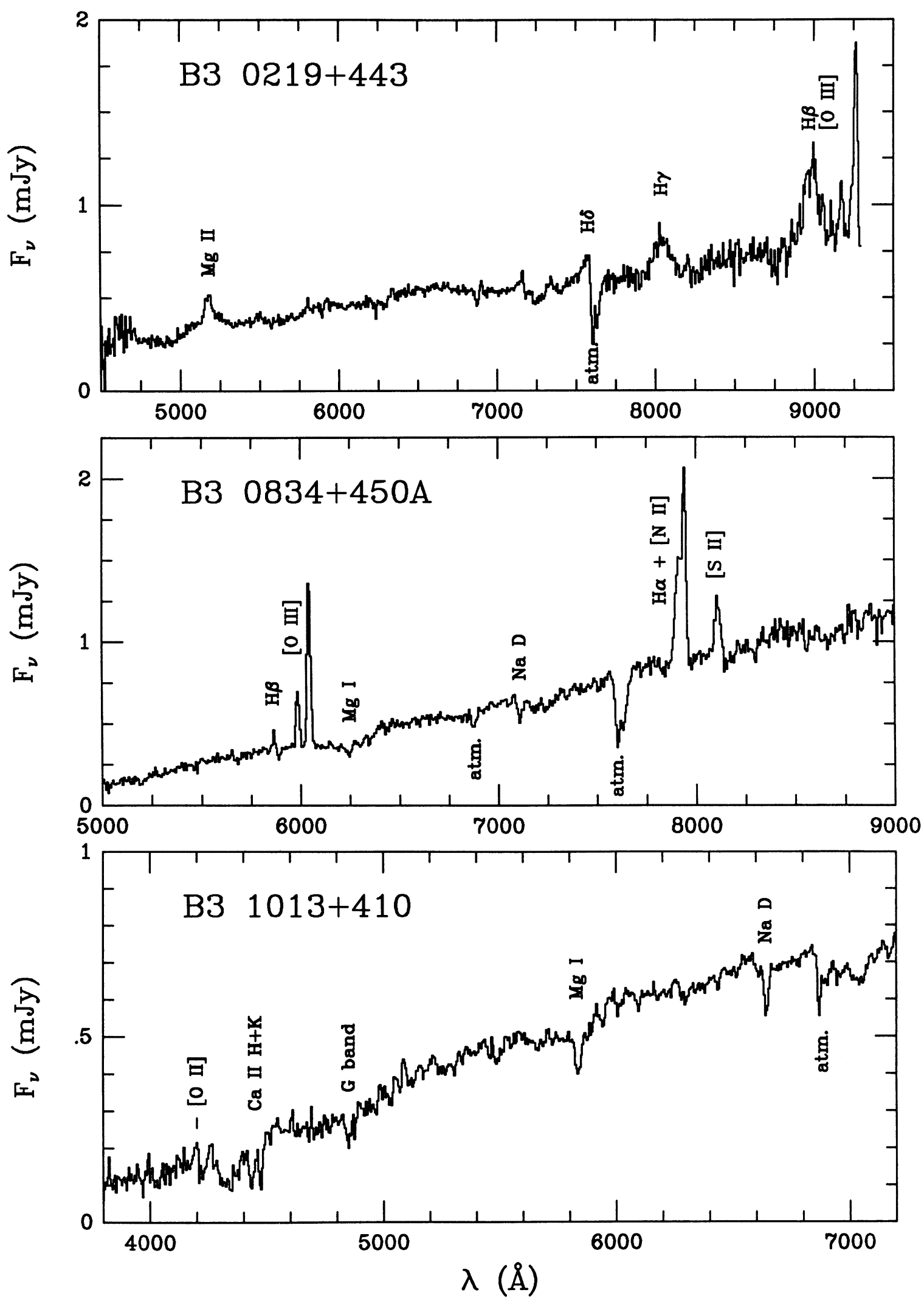

FIG. 1-Spectra of three representative sources: a quasar (B3 0219+443), and AGN (B3 0834+450A), and a galaxy with little or no line emission (B3 $1013+410)$. Some line features are identified. The flux calibration zero point is uncertain by as much as 0.5 mag and is different for different objects. 
TABLE I

Obsarved Iines and Redshifts

\begin{tabular}{|c|c|c|c|c|c|c|}
\hline Sou: & 1 rce & Iine & rest & $a b s$ & $\mathbf{z}$ & Type, average z \\
\hline B3 & $0141+398$ & several & ... & ... & 0 & Foregr. star \\
\hline B3 & $0219+443$ & $\begin{array}{l}\text { Kg II } \\
\text { B gamma } \\
\text { B bata } \\
\text { [O III] } \\
\text { [O III] }\end{array}$ & $\begin{array}{l}2799 \\
4340 \\
4861 \\
4959 \\
5007\end{array}$ & $\begin{array}{l}5177 \\
8041 \\
8987 \\
9174 \\
9261\end{array}$ & $\begin{array}{l}0.8496 \\
0.8526 \\
0.8487 \\
0.8500 \\
0.8497\end{array}$ & $\begin{array}{l}\text { Qso } \\
\langle z\rangle=0.850+-0.001\end{array}$ \\
\hline B3 & $0729+391$ & $\begin{array}{l}\text { Mg II } \\
\text { H bota } \\
{[0 \text { III] }}\end{array}$ & $\begin{array}{l}2799 \\
4861 \\
5007\end{array}$ & $\begin{array}{l}4651 \\
8093 \\
8328\end{array}$ & $\begin{array}{l}0.6616 \\
0.6648 \\
0.6633\end{array}$ & $\begin{array}{l}\text { Qso } \\
\langle z\rangle=0.663+-0.001\end{array}$ \\
\hline B3 & $0812+382$ & $\begin{array}{l}\text { Mg I blend } \\
\text { Na D }\end{array}$ & $\begin{array}{l}\mathbf{5 1 7 0} \\
\mathbf{5 8 9 0}\end{array}$ & $\begin{array}{l}6077 \\
6915\end{array}$ & $\begin{array}{l}0.1754 \\
0.1740\end{array}$ & $\begin{array}{l}\text { Galaxy } \\
\langle z\rangle=0.1747+-0.0007\end{array}$ \\
\hline B3 & $0834+450 A$ & $\begin{array}{l}\text { B bata } \\
\text { [O III] } \\
\text { [O III] } \\
\text { B alpha }\end{array}$ & $\begin{array}{l}4861 \\
4959 \\
5007 \\
6563\end{array}$ & $\begin{array}{l}5864 \\
5986 \\
6043 \\
7940\end{array}$ & $\begin{array}{l}0.2064 \\
0.2071 \\
0.2070 \\
0.2099\end{array}$ & $\begin{array}{l}\text { Seyfert } 2 \\
\langle z\rangle=0.2075+-0.001\end{array}$ \\
\hline B3 & $0836+402$ & 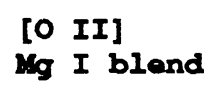 & $\begin{array}{l}3727 \\
5170\end{array}$ & $\begin{array}{l}4575 \\
6370\end{array}$ & $\begin{array}{l}0.2275 \\
0.2321\end{array}$ & $\begin{array}{l}\text { Galaxy } \\
\langle z\rangle=0.230+-0.002\end{array}$ \\
\hline B3 & $0906+421$ & $\begin{array}{l}\text { Mg I blend } \\
\text { Na D }\end{array}$ & $\begin{array}{l}\mathbf{5 1 7 0} \\
\mathbf{5 8 9 0}\end{array}$ & $\begin{array}{l}5895 \\
6715\end{array}$ & $\begin{array}{l}0.1402 \\
0.1401\end{array}$ & $\begin{array}{l}\text { Galaxy } \\
\langle z\rangle=0.1401+-0.0002\end{array}$ \\
\hline B3 & $0918+444$ & 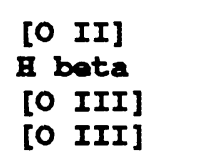 & $\begin{array}{l}3727 \\
4861 \\
4959 \\
5007\end{array}$ & $\begin{array}{l}4647 \\
6112 \\
6177 \\
6242\end{array}$ & $\begin{array}{l}0.2468 \\
0.2574 \\
0.2456 \\
0.2468\end{array}$ & $\begin{array}{l}\text { Seyfert } 2 \\
\langle z\rangle=0.248+-0.002\end{array}$ \\
\hline B3 & $1013+410$ & $\begin{array}{ll}{[0} & I I] \\
\text { Mg I blend } \\
\text { Na } & D\end{array}$ & $\begin{array}{l}3727 \\
5170 \\
5890\end{array}$ & $\begin{array}{l}4194 \\
5835 \\
6642\end{array}$ & $\begin{array}{l}0.1253 \\
0.1286 \\
0.1277\end{array}$ & $\begin{array}{l}\text { Galaxy } \\
\langle z\rangle=0.127+-0.0015\end{array}$ \\
\hline B3 & $1141+374(\mathrm{~N})$ & $\begin{array}{l}\text { Mg I blend } \\
\text { Na D } \\
\text { B alpha }\end{array}$ & $\begin{array}{l}5170 \\
5890 \\
6563\end{array}$ & $\begin{array}{l}5775 \\
6575 \\
7144\end{array}$ & $\begin{array}{l}0.1170 \\
0.1163 \\
0.0886\end{array}$ & $\begin{array}{l}\text { Interacting Double } \\
\langle z\rangle=0.1165+-0.0005\end{array}$ \\
\hline B3 & $1141+374$ (s) & 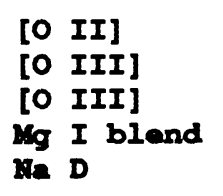 & $\begin{array}{l}3727 \\
4959 \\
5007 \\
5170 \\
5890\end{array}$ & $\begin{array}{l}4150 \\
5525 \\
5583 \\
5765 \\
6565\end{array}$ & $\begin{array}{l}0.1135 \\
0.1142 \\
0.1151 \\
0.1151 \\
0.1146\end{array}$ & $\begin{array}{l}\text { Interacting Double } \\
\text { Seyfert } 2 ? \\
\langle z\rangle=0.1145+-0.0005\end{array}$ \\
\hline B3 & $1309+412 \mathrm{~A}$ & $\begin{array}{l}\text { [O III] } \\
\text { [O III] } \\
\text { Mg I blend } \\
\text { Na D } \\
\text { H alpha } \\
\text { [N II] }\end{array}$ & $\begin{array}{l}4959 \\
5007 \\
5170 \\
5890 \\
6563 \\
6583\end{array}$ & $\begin{array}{l}5507 \\
5559 \\
5740 \\
6540 \\
7284 \\
7307\end{array}$ & $\begin{array}{l}0.1105 \\
0.1102 \\
0.1103 \\
0.1104 \\
0.1099 \\
0.1099\end{array}$ & $\begin{array}{l}\text { Seyfert } 2 \\
\langle z\rangle=0.1103+-0.0002\end{array}$ \\
\hline
\end{tabular}

but were unusable for the redshift measurements due to blending and ill-defined central wavelengths. In some cases (e.g., B3 1309+412A) the $\mathrm{H} \alpha$ and [N II] lines gave somewhat discrepant redshifts from the remaining lines; this is probably an artifact of line blending and/or poor wavelength calibration near the CCD edges.

B3 $0219+443$ is a steep radio spectrum quasar $(\alpha=$ $-1.17)$, and as such is worthy of further attention. B3 
$1141+374$ is identified with a close double, both components of which show some line emission. The VLA map published by Vigotti et al. (1989) shows distorted and diffuse radio emission. The line emission is stronger in the southern component, and the $\mathrm{H} \beta /[\mathrm{O}$ III] ratio is characteristic of a high-ionization object, e.g., Seyfert 2 nucleus. It is possible that the interaction of two galaxies is the cause for the nuclear activity in one of them.

We are indebted to the staff of Palomar Observatory for their expert help and, in particular, to Juan Carrasco, Dave Tennant, John Henning, and Skip Staples. We also thank Helen Johnston, Alain Picard, and Georges Meylan for their assistance with the observations. This work was supported in part by the Alfred P. Sloan Foundation and the California Institute of Technology (S.D.) and by a Caltech Institute Fellowship (D.J.T.).

\section{REFERENCES}

Ficarra, A., Grueff, G., and Tomassetti, G. 1985, Astr. Ap. Suppl., 59, 255.

Oke, J. B., and Gunn, J. E. 1982, Pub. A.S.P., 94, 586. . 1983, Ap. J., 266, 713.

Vigotti, M., Grueff, G., Perley, R., Clark, B., and Bridle, A. 1989, A.J., 98, 419. 